\title{
Allogeneic Glioblastoma Stem-like Cell Line Lysate-pulsed Autologous Dendritic Cell Vaccine
}

National Cancer Institute

\section{Source}

National Cancer Institute. Allogeneic Glioblastoma Stem-like Cell Line Lysate-pulsed Autologous Dendritic Cell Vaccine. NCI Thesaurus. Code C113296.

A cell-based cancer vaccine composed of autologous dendritic cells (DCs) pulsed with lysates from an allogeneic glioblastoma (GBM) stem-like cell line, with potential immunostimulatory and antineoplastic activities. Upon administration allogeneic glioblastoma stem-like cell line lysate-pulsed autologous dendritic cell vaccine exposes the immune system to GBM stem cell antigens, which may result in cytotoxic T lymphocyte (CTL) and antibody responses against GBM cells. This leads to GBM cell lysis. GBM stem-like cells contain a specific range of antigens that are essential for the neoplastic growth and survival of GBM cells. 